\title{
Laser cleaning of particles from silicon wafers: capabilities and mechanisms J. Graf ${ }^{1, a}$, F. Lang ${ }^{1}$, M. Mosbacher ${ }^{1}$ and P. Leiderer ${ }^{1}$ \\ ${ }^{1}$ University of Konstanz, Department of Physics, Fach M676, D-78457 Konstanz, Germany \\ a johannes.graf@uni-konstanz.de
}

Keywords: particle removal; laser cleaning; dry laser cleaning; steam laser cleaning.

\section{Introduction}

The preparation of surfaces free of particle contamination is one of the crucial prerequisites for a further increase in the integration density of ICs and for the progress in nanotechnology. Therefore the removal of sub-micron sized particles from silicon wafers is of great interest. For this purpose a variety of cleaning methods is currently under investigation.

In semiconductor industry an ideal cleaning technique should be capable of removing particles with a diameter considerably below $100 \mathrm{~nm}$, while a damage to the substrate has to be strictly avoided. Furthermore the process should be environmentally friendly and cost-effective. A promising approach which meets these requirements is called Laser Cleaning. So far two major approaches can be distinguished. The first one is called Dry Laser Cleaning (DLC), where the surface to be cleaned is simply irradiated by a short laser pulse [1,2,3]. In Steam Laser Cleaning (SLC) a thin layer of a liquid energy transfer medium is deposited on the surface prior to the laser pulse $[1,2,4,5]$.

In this paper we will present a short overview of these techniques followed by some of our recent results. The emphasis lies on the underlying mechanisms and an industrial applicability of both cleaning methods.

\section{Experimental aspects}

In our experiments we used commercial available polystyrene (PS; Interfacial Dynamics Corporation, Portland, OR, USA) and silica particles $\left(\mathrm{SiO}_{2}\right.$; Bangs Laboratories Inc., Fishers, IL, USA and Duke Scientific Corp., Palo Alto, CA, USA) with diameters in the range of $50 \mathrm{~nm}$ to $2000 \mathrm{~nm}$. Their spherical shape and small size distribution facilitates comparisons with theoretical models and investigations of size dependent effects. Industrial silicon (100) wafers (Wacker Siltronic, Burghausen, Germany), cleaned in isopropyl alcohol (IPA) in an ultrasonic bath, were used as substrates. The particles were applied on precleaned wafers by a spin coating technique. Unless otherwise noted a frequency doubled, Q-switched Nd:YAG laser (FWHM = 9 ns) was used. After the cleaning procedure the cleaned areas have been characterized by atomic force microscopy for damage inspection.

\section{Dry Laser Cleaning (DLC)}

Already in the first publications on DLC a physical mechanisms responsible for particle removal was suggested [1,2]. The removal process was attributed to the thermoelastic expansion due to the absorbed energy and the associated deceleration of the substrate surface [6]. The particles leave the surface during the deceleration process due to inertia forces. The decelerations for a typical laser cleaning fluence have been estimated to be on the order of $10^{7} \mathrm{~m} / \mathrm{s}^{2}$, just the order of magnitude that is thought to be necessary for removal of such small particles [2]. This order of magnitude has been experimentally verified by V. Dobler et al. [7] by measuring the deceleration of an silicon surface after pulsed laser irradiation was measured by a heterodyne interferometer setup.

Unfortunately thermal expansion is not the only effect that occurs during the DLC process. An inspection by scanning electron microscopy (SEM) or atomic force microscopy (AFM) of samples cleaned by DLC reveals damages all over the cleaned areas. They manifest as melting pools or even 
holes in the surface, typical examples can be seen in Figure 1. It can be shown that these defects are localized exactly at the former particle positions $[8,9,10]$.

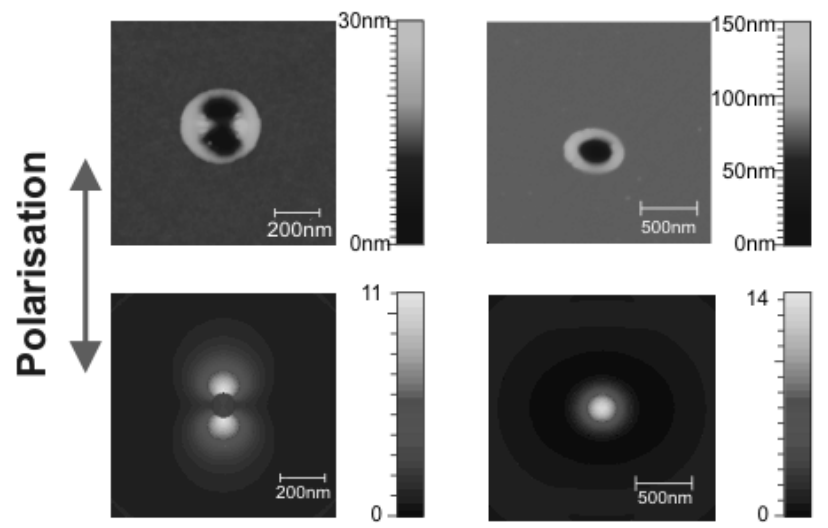

Figure 1 : AFM images of surface modifications created by near-field induced substrate ablation (top) and a comparison to the near-field distribution on the silicon surface calculated by the Mie theory (bottom). The particle sizes were $320 \mathrm{~nm}$ (left) and $800 \mathrm{~nm}$ (right) with $800 \mathrm{~nm}$ laser wavelength. Note the good agreement between the calculations and the experimental ablation pattern.

Such defect formations can be attributed to a focussing of the laser irradiation at the particles. Depending on the particle size the intensities in the near-field of the particles can exceed the incoming laser intensity by several ten times and can lead to local melting, ablation and even plasma formation in the optical near-field of the particles [10]. Since the particle diameters are comparable or below the applied laser wavelength this effect is described by the Mie theory [11]. The relevant variable of this theory is the so called size parameter

$$
x=\frac{\pi \cdot d}{\lambda}
$$

with particle diameter $d$ and laser wavelength $\lambda$. But the Mie theory not only describes an enhancement of the laser intensity in the particles' near-field, it also predicts that for certain values of the size parameter the enhancement should be particularly efficient, resulting in a resonant intensity enhancement, the so-called "Mie-resonances". Strictly speaking this theory is only valid for spherical particles in homogenous surroundings and is thus neglecting any influence of the substrate. However, more advanced theories, taking into account the influence of the substrate, confirm the results of Mie's theory as far as the intensity enhancement and the existence of resonances are concerned. In comparison to the simpler Mie approach, however, these resonances are much more pronounced and may be located at different values of the size parameter [6].

If particle removal in DLC is dominated by local ablation underneath the particles rather than thermal expansion it should give rise to a resonant, non-monotonous dependence of the laser fluence necessary for particle removal on the size parameter. Indeed this prediction can be verified experimentally:

In Figure 2 the cleaning thresholds for the removal of PS-particles of different particle sizes are plotted for two different laser systems, a Q-switched Nd:YAG laser ( $\lambda=532 \mathrm{~nm}, \mathrm{FWHM}=9 \mathrm{~ns})$ and a Ti:Sa-femtosecond laser system $(\lambda=800 \mathrm{~nm}, \mathrm{FWHM}=200 \mathrm{fs})$.

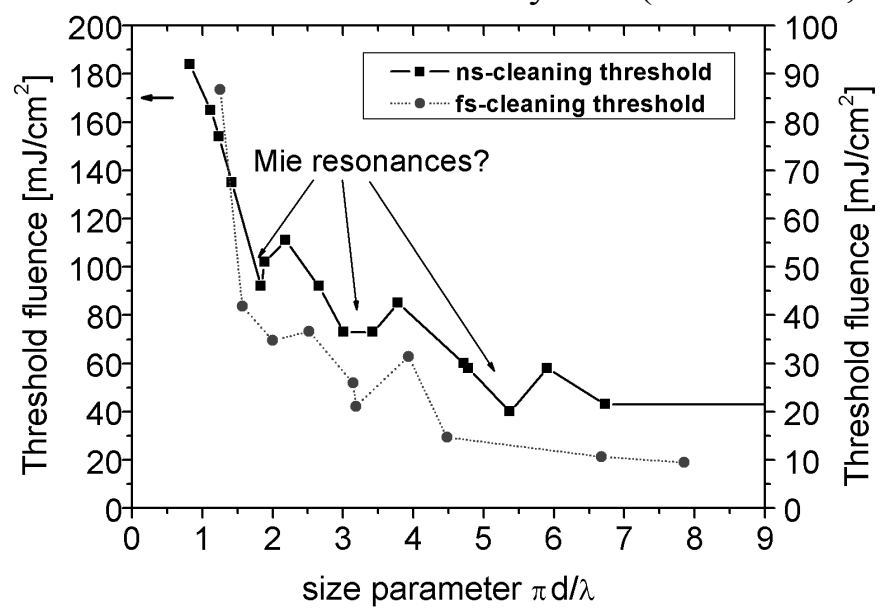

Figure 2 : Plotted are the laser fluences necessary for particle removal as a function of the size parameter. The two curves correspond to two different laser systems: a frequency doubled $\mathrm{Nd}$ :YAG $(\lambda=532 \mathrm{~nm}$, FWHM $=9 \mathrm{~ns})$ and a Ti:Sa- fs - System $(\lambda=800 \mathrm{~nm}$, FWHM $=200 \mathrm{fs})$. The minima in both curves at the same size parameters might correspond to Mieresonances. 
Since the wavelength of the used laser systems is different, the cleaning threshold values are plotted versus the size parameter $x$. For three values of this parameter a minimum in the laser fluence necessary for particle removal can be seen, that might correspond to Mie-resonances. Due to the discrete steps of available particle sizes in this experiments the density of data points is too low to manifest this behaviour. As the size parameter not only depends on the particle size, but also on the applied wavelength, a variation of the latter will also result in a variation of $x$. Therefore we used an optical parametric oscillator $(\mathrm{OPO}, \mathrm{FWHM}=9 \mathrm{~ns})$ and varied the wavelength of the applied laser pulses between $500 \mathrm{~nm}$ and $580 \mathrm{~nm}$. The results are shown in Figure 3. On the left hand side of the diagram we show the raw data for three different particle sizes. To draw conclusions on the influence of the near-field intensities on the DLC cleaning process the data again have to be plotted over the size parameter $x$, but also the wavelength dependent reflectivity of the substrate has to be compensated. Local ablation takes place in the top few $\mathrm{nm}$ of the sample. So the amount of energy deposited in a shallow layer at the silicon surface is the crucial parameter for the local substrate ablation. Therefore the raw data were furthermore corrected by the wavelength dependent optical penetration depth. The result of this is shown on the right hand side of Figure 3 . The continuous behaviour of the cleaning threshold for different particle sizes justifies the assumption, that the energy density close to the surface is the relevant parameter for particle removal. The nonmonotonous trend strengthens the assumption that the near-field induced ablation
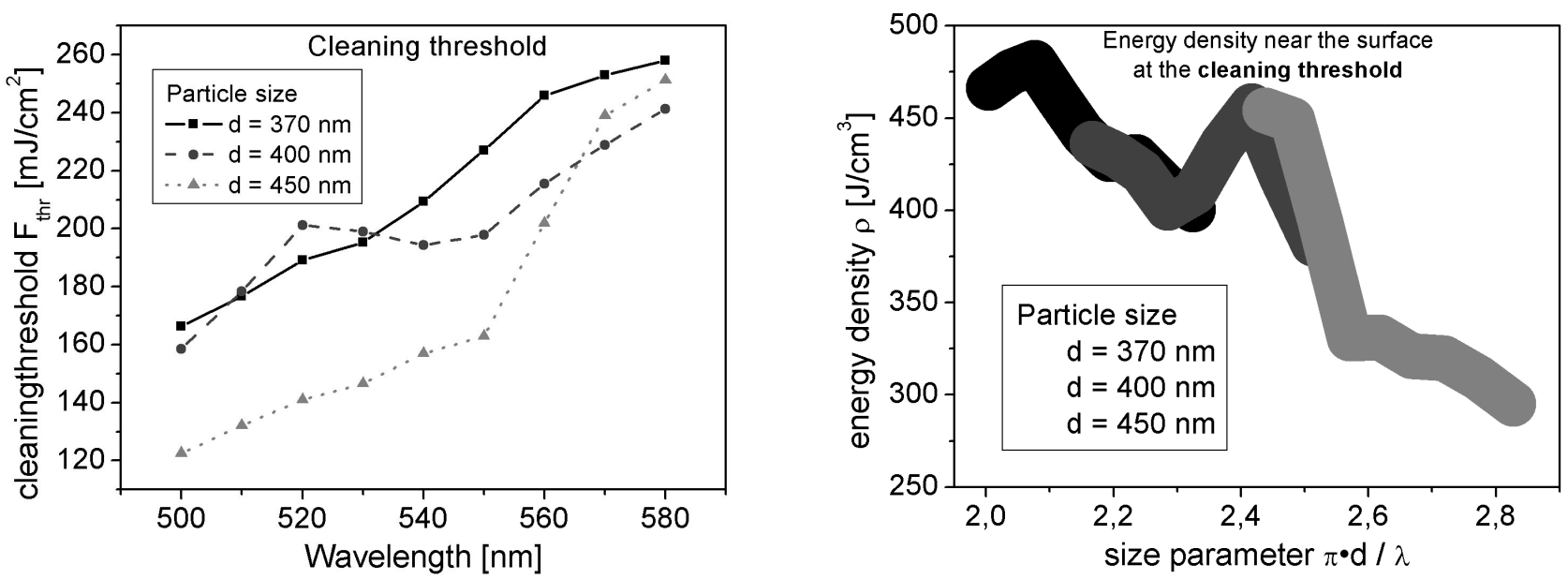

Figure 3 : left: measured cleaning thresholds for three different particle sizes versus the incident wavelength of a nsOPO; right: After correction for the optical properties of the substrate (details see text) the data of the left diagram are plotted versus the size parameter. The line thickness represents the experimental error both in size parameter as well as in energy density.

plays a major role in the particle removal process. Besides the two mechanisms discussed above several more physical mechanisms may contribute to particle removal in DLC. But against the background of the fact, that for all parameters investigated so far particle removal was always accompanied by surface modifications, we conclude, that local ablation constitutes the dominant mechanism in DLC.

Since substrate modification is unacceptable for any industrial application, different attempts have been made to avoid such damage by near-field focusing effects in a DLC process, unfortunately so far without success. A more promising technique with respect to this is described in the following.

\section{Steam Laser Cleaning (SLC)}

This laser cleaning method involves a thin layer of a liquid energy transfer medium, in most cases a water alcohol mixture [1,2,5], deposited on the surface prior to the laser pulse. The pressure wave generated during the explosive evaporation of the energy transfer medium serves as an additional cleaning mechanism. Since the force exerted on the particle by the pressure wave exceeds the 
adhesion forces for all particle sizes we have measured (diameter 50-800 nm), the laser fluence necessary for particle removal becomes independent of the particle size and material [12], and is then given by the threshold for bubble nucleation of the energy transfer medium [13]. This universal cleaning threshold is an important advantage with respect to possible applications. Furthermore the adhesion forces between the particles and the substrate are reduced and the near field enhancement at the particles is less pronounced due to the better matching between the particles' dielectric constants and their surrounding. Unfortunately the particle removal [13] as well as the underlying physical processes [14] are quite sensitive to the thickness and composition of the energy transfer layer. However we could demonstrate, that by adjusting the parameters of the process properly particles with diameters down to at least $60 \mathrm{~nm}$ can be removed without damaging the substrate [12].

\section{Conclusion}

In this paper we showed that in Dry Laser Cleaning for all conditions investigated so far the local substrate ablation due to near-field enhancement at the particles is the dominant mechanism for particle removal. Thus cleaning is always accompanied by a local damages of the substrate. This process is therefore not suitable for industrial application, although most of the publications on laser assisted particle removal focus on this cleaning strategy. A more promising method with respect to this is the so called Steam Laser Cleaning. By adjusting the process parameters carefully the removal of particles down to at least $60 \mathrm{~nm}$ in diameter is possible, without damaging the silicon surface.

\section{References}

[1] W. Zapka, W. Ziemlich and A.C. Tam, Appl. Phys. Lett. 58, (1991), p. 2217.

[2] A. C. Tam, W. P. Leung, W. Zapka and W. Ziemlich, J. Appl. Phys. 71 (1992), p. 3515.

[3] A. C. Engelsberg, Mater. Res. Soc. Symp. Proc. 315 (1993), p. 255.

[4] I. V. Beklemyshev, V. V. Makarov, I. I. Makhonin, Y. N. Petrov, A. M. Prokhorov and V. I. Pustovoi, JETP Lett. 46 (1987),p. 347-350.

[5] K. Imen, J. Lee and S.D. Allen, Appl. Phys. Lett. 58 (1991), p. 203-205.

[6] B. S. Luk'yanchuk, M. Mosbacher, Y. W. Zheng, H. -J. Münzer, S. M. Huang, M. Bertsch, W. D. Song, Z. B. Wang, Y. F. Lu, O. Dubbers, J. Boneberg, P. Leiderer, M. H. Hong, T. C. Chong, in Laser Cleaning (2002).

[7] V. Dobler, R. Oltra, J.P. Boquillon, M. Mosbacher, J. Boneberg and P. Leiderer, Appl. Phys. A 69 (1999), p. 335.

[8] P. Leiderer, J. Boneberg, V. Dobler, M. Mosbacher, H.-J. Münzer, N. Chaoui, J. Siegel, J. Solis, C.N. Afonso, T. Fourrier, G. Schrems and D. Bäuerle, Proc. SPIE 4065 (2000), p. 249-259.

[9] M. Mosbacher, H.-J. Münzer, J. Zimmermann, J. Solis, J. Boneberg, P. Leiderer, Appl. Phys. A 72 (2001), p. 41.

[10] H.-J. Münzer, M. Mosbacher, M. Bertsch, J.Zimmermann, P. Leiderer, J. Boneberg, J. Microscopy 202 (2001), p. 129.

[11] G. Mie, Ann. Physik 4 (1908), p. 377-445.

[12] M. Mosbacher, V. Dobler, J. Boneberg and P. Leiderer, Appl. Phys. A 70 (2000), p. 669.

[13] F. Lang, M. Mosbacher and P. Leiderer, Applied Physics A 77 (2003), p. 117-123.

[14] F. Lang, S. Georgiou and P. Leiderer, Appl. Phys. Lett., accepted for publication. 